\title{
Abundância e sazonalidade de histerídeos (Coleoptera) associados ao esterco de granja aviária da Região Nordeste do Estado de São Paulo, Brasil
}

\author{
Welber Daniel Zanetti Lopes ${ }^{1}$, Wilton Carlos Zanetti Lopes², Fábio Habermann da Costa ${ }^{3}$, Júlio César de \\ Carvalho Balieiro ${ }^{4}$ A Ângelo Pires do Prado 5
}

${ }^{1}$ Departamento de Medicina Veterinária Preventiva, Faculdade de Ciências Agrárias e Veterinária, Via de acesso prof. Paulo Donatto Castellani, s/n, 14884-900, FCAV/UNESP, Jaboticabal-SP, Brasil.wdzlopes@ fcav.unesp.br

${ }^{2}$ Departamento de Biologia Celular e Molecular e Bioagentes Patogênicos, Faculdade de Medicina, Ribeirão Preto, Universidade de São Paulo, Av. Bandeirantes 3900, 14049-900, Ribeirão Preto, SP, Brasil

${ }^{3}$ Médico Veterinário Graduado na Faculdade de Medicina Veterinária-UNIFEOB, São João da Boa Vista-SP, Brasil.

${ }^{4}$ Faculdade de Zootecnia e Engenharia de Alimentos da Universidade de São Paulo, Pirassununga-SP, Brasil.

${ }^{5}$ Departamento de Parasitologia, Instituto de Biologia-UNICAMP, Campinas-SP.

\begin{abstract}
Abundance and seasonality of Histeridae (Coleoptera) associated to brooding hen excrement in the northeast region of the state of São Paulo, Brazil. Programs for biological control are usually based on the release of predators and parasites so that no hazard is posed to the natural environment. Coleoptera of the Histeridae family feed on the larvae of synantropic diptera, enabling their use in the control of such flies. The objective of this work was to carry out a survey of the Histeridae species present at a poultry house situated in the city of São João da Boa Vista, state of São Paulo, Brazil, aiming to describe their seasonality and the possible associations between the adult specimens. Samples were collected from the poultry houses from January 2001 to December 2002, using two collection methods: soil traps and Berlese funnel. The following Histeridae species were encountered: Euspilostus modestus, Euspilostus spp., Carcinops troglodytes and Hololepta quadridentata. The number of specimens found for each species depended on the collecting method. In the case of the soil trap, E. modestus corresponded to $87.01 \%$ of the total number of collected individuals. On Berlese funnel, method C. troglodytes corresponded to $84.38 \%$ of the total number of collected beetles. The presence of $E$. modestus and $H$. quadridentata was significantly correlated to climatic variations, and positively especialy rainfall ( $\mathrm{P}<0.05$ and $\mathrm{P}<0.01$ for E. modestus and H. quadridentata, respectively).
\end{abstract}

KEYWORDS. Carcinops troglodytes; Eupilostus modestus; natural enemies; poultry house.

RESUMO. Abundância e sazonalidade de histerídeos (Coleoptera) associados ao esterco de granja aviária da Região Nordeste do Estado de São Paulo, Brasil. Programas que visam o controle biológico precisam ser embasados principalmente em liberações de predadores e parasitóides para tentar minimizar efeitos adversos para o ambiente. Os coleópteros da família Histeridae como predam larvas de dípteros sinantrópicos podem auxiliar no controle das moscas que se desenvolvem em fezes acumuladas. Este trabalho teve como objetivo realizar um levantamento das espécies de histerídeos em granja avícola no município de São João da Boa Vista, estado de São Paulo, verificar seu padrão sazonal de ocorrência e investigar possíveis associações entre as mesmas. As coletas foram realizadas de janeiro de 2001 a dezembro de 2002 , utilizando-se dois métodos de coleta (armadilhas de solo e o funil de Berlese - Tullgren). As espécies de histerídeos encontradas foram: Euspilostus modestus, Euspilostus (Hesperosaprinus) spp., Carcinops troglodytes e Hololepta quadridentata. O número de cada espécie diferiu entre os métodos utilizados. Na armadilha de solo, E. modestus correspondeu a $87,01 \%$ do número total de indivíduos capturados, enquanto que no funil, $C$. troglodytes obteve maior destaque $(84,38 \%)$. E. modestus e H. quadridentata apresentaram padrões de variação mensal positivamente correlacionada com a precipitação pluviométrica $(\mathrm{P}<0,05$ e $\mathrm{P}<0,01$, respectivamente).

PALAVRAS-CHAVE. Carcinops troglodytes; Eupilostus modestus; Granja Avícola; Inimigos Naturais.

Desde que o homem começou a confinar animais, o controle dos artrópodes tem sido um constante desafio, além disto, o uso indiscriminado de inseticidas químicos geralmente utilizados nos programas de controle destas pragas polui o ambiente, contamina carnes, ovos e leite, e agrava ainda mais o problema nas criações. Legner \& Brydon (1966) e Legner \& Olton (1968) relatam que, ao se erradicar os dípteros das áreas de criação animal, obtém-se o benefício tanto na produção, como no consumo. No entanto, é essencial que o controle químico seja feito como complemento do método biológico (Guimarães 1985). O uso de inseticidas químicos são muitas vezes necessário em situações críticas, porém de forma limitada, uma vez que tais produtos, exercem efeito tóxico nos controladores naturais de moscas (predadores e parasitóides).
Outro fator a acrescentar é o rápido desenvolvimento de resistência aos inseticidas pelo díptera Musca domestica (Linnaeus 1758) e sendo assim, poucos produtos estão disponíveis para seu controle.

Programas que visam o controle biológico aplicado e/ou clássico de pragas precisam ser, via de regra, embasados em liberações de predadores, parasitóides e competidores, em larga escala, ou seja, inimigos naturais eficientes que não acarretem em conseqüências adversas para o ambiente (Carraro \& Azevedo 2000).

Por estas razões, durante as últimas décadas têm sido desenvolvido novos métodos de controle que são ecológicos (Mijares et al 2000).

Uma galinha de $1,8 \mathrm{Kg}$ em produção, produz por dia, $113 \mathrm{~g}$ 
de fezes úmidas as quais servem como meio para o desenvolvimento de inúmeras espécies de artrópodes, e dentre esses, os dípteros muscóideos sinantrópicos que desempenham importante papel na baixa sanitização das criações. Representantes como a Musca domestica e Chrysomya putoria (Wiedemann, 1918) desenvolvem-se muito bem neste substrato e são importantes veiculadoras de agentes patogênicos, tanto para as aves quanto para o homem (North \& Bell 1990). A abundância de tais dípteros, em locais de acúmulo de esterco de aves, atrai a presença de muitos inimigos naturais, que atuam como seus controladores naturais, (Gianizella \& Prado 1998).

Os Histeridae apresentam hábitos predatórios, tanto na fase larval, quanto na adulta. Buscam seus alimentos, principalmente, em ambientes em decomposição, onde ao encontrarem larvas de primeiro e segundo ínstares de dípteros sinantrópicos, atuam como predadores ou como agentes de "manejo físico". Desta forma, ocorrem em fezes animais, auxiliando no controle de moscas de importância médicoveterinária que aí se desenvolvem (Clark 1895; Peck \& Anderson 1969; Rodrigues \& Marchini 1998).

Portanto é essencial que se realize o levantamento e a identificação das espécies de inimigos naturais em um determinado ambiente de criação animal, a fim de orientar melhor o controle dos dípteros por meio de métodos integrados (Georghiom et al 1967; Taylor 1982; Imai 1987).

$\mathrm{O}$ trabalho teve como objetivos realizar um levantamento qualitativo e quantitativo das espécies de histerídeos, verificar sua sazonalidade, e investigar possíveis associações entre os adultos, visando uma possível utilização no controle biológico.

\section{MATERIALE MÉTODOS}

Descrição da Granja: As coletas foram realizadas na granja Crisdan, situada a $7 \mathrm{Km}$ do município de São João da Boa Vista, estado de São Paulo (22 $01^{\circ}$ ' S, $046^{\circ} 48^{\prime}$ W, altitude de 763 metros).

A granja considerada de pequeno porte é constituída por dois conjuntos de quatro galpões (abertos) cada, e tem capacidade para alojar 25.000 galinhas, das linhagens "Hy Line" e "Hy-line Brown", em fase de postura.

No galpão de coleta estiveram dispostos três conjuntos de gaiolas separados por dois corredores de concreto de $0,5 \mathrm{~m}$ de largura. As fileiras das gaiolas estão distribuídas em degrau, ou seja, uma fileira mais interna a $0,5 \mathrm{~m}$ do chão, e uma mais externa a $1 \mathrm{~m}$ (tipo "narrow house"). As gaiolas variam de tamanho, podendo abrigar de 2 a 4 galinhas. Sob as gaiolas, o chão é de terra onde as fezes se acumulam.

Ao redor da granja havia cultura agrícola e durante o período analisado, a predominância foi de café e milho. Durante a coleta, nenhum inseticida foi utilizado sobre o esterco, porém o mesmo foi retirado em 24/04 e 20/09 de 2001 e em 15/03 e 02/ 11 de 2002. Devido as altas precipitações constatadas no verão, o uso de óxido de cálcio e de serragem foi relativamente comum.

Foram realizadas setenta e cinco coletas entre 17/01/2001 a
26/12/2002, com intervalos de sete dias, durante os primeiros doze meses, passando a quinzenais nos demais meses, totalizando 1.125 armadilhas e $300 \mathrm{Kg}$ de esterco. Para amostragem dos espécimes adultos foram utilizados 2 métodos distintos; armadilha-de-solo (método 1) e o funil de BerleseTullgren (método 2).

Método 1 - Armadilha-de-solo. Estas armadilhas foram utilizadas visando a obtenção de histerídeos adultos (Walker 1985; Sunmerlin 1989). Consistiu na utilização de frascos plásticos com $9,0 \mathrm{~cm}$ de altura e $8,0 \mathrm{~cm}$ de diâmetro contendo $200 \mathrm{ml}$ de um líquido conservante e fixador (80\% de água; 5\% de glicerina; $5 \%$ de álcool a 70\%; 5\% de formol e 5\% de detergente) para posterior identificação e quantificação em microscópio esterioscópio. À cada coleta as armadilhas foram retiradas e substituídas por outras com novo fixador. As armadilhas, numeradas de 1 a 15 , foram escolhidas aleatoriamente e enterradas sob as gaiolas, ao longo de três corredores (5 armadilhas/corredor) A, B e C, próximo ao esterco acumulado.

A distribuição das armadilhas foi feita por "amostragem sistemática", que consistiu em delimitar áreas em quadrados contíguos $\left(1 \mathrm{~m}^{2}\right)$, onde as mesmas foram distribuídas (Krebs 1989).

Método 2 - Extração direta dos espécimes do esterco. $O$ esterco acumulado sob as gaiolas, à observação visual, apresentava, aproximadamente, cinco tipos distintos de consistência: líquido, pastoso, pastoso-firme, firme e seco. No galpão havia esterco com uma ou mais consistências diferentes. As coletas das amostras foram dirigidas de forma a obter esterco de todas as consistências.

Na coleta das amostras de esterco foi utilizada uma pá de lixo que facilitava o recolhimento do esterco acumulado, ao invés da introdução de um cilindro de metal, como recomendado por Peck \& Anderson (1970). Para obtenção deste material foram extraídas amostras aleatórias num total de aproximadamente $4 \mathrm{Kg}$ de esterco por coleta.

Neste método foi utilizado funil de Berlese-Tullgren, em número de seis. Cada funil consistiu de um tubo feito de papel cartolina sobre um vidro, com líquido fixador e conservador (álcool a 70\%). Sobre o funil foi fixada uma tela de malha de $1 \mathrm{~cm}$. O esterco era colocado dentro da lata e ficava exposto a lâmpada de $25 \mathrm{~W}$, durante 5 dias.

Para identificação dos histerídeos adultos, foram utilizados os trabalhos de Hinton (1945); Wenzel (1955); Borror (1970) e Arnett (1973). O material testemunho encontra-se na Coleção Entomológica da Faculdade de Medicina Veterinária do Centro Universitário das Faculdades Integradas da Fundação de Ensino "Octávio Bastos".

Análises estatísticas. Foram verificadas as principais fontes de variações que poderiam influenciar no número de coleópteros (por gênero e/ou espécie), avaliando-se as significâncias dos efeitos de coleta, local, mês e ano, além dos efeitos de temperatura, umidade relativa do ar e precipitação 
pluviométrica incluídas como covariáveis. Nas análises estatísticas, adotou-se a transformação de escala raiz quadrada no número total de histerídeos capturados mais um (por gênero e/ou espécie) (Banzatto \& Kronca 1989). As análises finais, contemplando somente as fontes de variações significativas foram descritas por meio de um modelo que considerou os efeitos de mês e ano de captura, além da covariável linear pluviosidade mensal, ou seja:

$$
y_{i j k}=\mu+M_{i}+A_{j}+b_{1}\left(P l u v_{i j k}-\overline{P l u v}\right)+e_{i j k}
$$

em que: $y_{i j k}$ é o valor transformado para escala raiz quadrada do número total de histerídeos capturados mais um no mês i, do ano j; $M_{i}$ é o efeito do i-ésimo mês de captura; $A_{j}$ é o efeito do j-ésimo ano de captura; $b_{1}$ é o coeficiente de regressão linear da característica $y_{i j k}$ em relação à pluviosidade; $P l u v_{i j k}$ é a pluviosiodade no ano j e no mês i incluída no modelo como covariável; $\overline{P l u v}$ é a média da pluviosidades observadas durante o estudo; $\mathrm{e}_{i j k}$ é o efeito aleatório residual associado às observações, no ano j e no mês i, assumido com média 0 e variância $\sigma_{e}^{2}$.

Face à utilização da covariável pluviometria (com efeito linear) ter apresentado resultados significativos para algumas espécies avaliadas, as análise das correlações momentoproduto de Pearson foram utilizadas para avaliação de quão associadas foram os números de coleópteros capturados e os índices pluviométricos. O teste para verificação de correlações realizada pelo programa supracitado, é um Teste $t$ de Student específico para correlações.

Para tanto, foram utilizados os procedimentos GLM do programa Statistical Analysis System, versão 6.12 (SAS 1995).

O teste de homogeneidade foi realizado segundo Fowler $\&$ Cochen (1990).

\section{RESULTADOSEDISCUSSÃO}

Foram coletados 89.044 Histeridae (Tabela I), pertencentes as espécies: Euspilostus modestus (Erichson, 1834), Euspilostus (Hesperosaprinus) spp., Carcinops troglodytes (Paykull, 1811) e Hololepta quadridentata (Fabricius, 1801).

O número e a proporção de cada espécie foi diferente quando comparado os métodos de coleta. Nas coletas com uso de funil de Berlese, foram capturados todas as espécies, totalizando 5,25\% dos histerídeos. C. troglodytes foi a espécie mais abundante, perfazendo 3.943 dos 4.673 coleópteros coletados $(84,38 \%)$ (Tabela I). Nas coletas feitas com o uso de armadilhas de solo também foram encontradas todas as espécies, mas o número de histerídeos obtido foi 18 vezes maior do que no método anterior, $(94,75 \%)$. E. modestus foi a espécie mais abundante, totalizando 73.415 dos 84.371 coleópteros capturados $(87,01 \%)$ (Tabela I).

Quando somados os dois métodos de coleta, E. modestus e C. troglodytes corresponderam a $98,53 \%$ de todos histerídeos coletados, evidenciando um alto grau de adaptação a este substrato artificial produzido em criação industrial de aves poedeiras.

Apesar destas duas espécies terem sido tão dominantes, o teste de homogeneidade (Fowler \& Cohen 1990) descartou a hipótese de que a diferença de abundância obtida entre as espécies nos diferentes métodos de coleta, tenha sido erro de amostragem. Portanto, supõem-se que os nichos espaciais existentes na granja Crisdan já estejam ocupados, e que esta diferença de prevalência possa estar associada a competência ecológica destas espécies no aproveitamento dos recursos oferecidos por aquele agroecossistema (Krebs 1989 e Gianizella \& Prado 1998).

Tabela I. Coleópteros histerídeos coletados por meio de armadilha de solo e funil de Berlese-Tullgren em granja avícola no estado de São Paulo, e dados sobre temperatura (TEMP) umidade relativa (UR), precipitação pluviométrica (PP), durante os meses de Janeiro de 2001 a Dezembro de 2002, número de coletas realizadas (N), média (MED), desvio padrão (DP), amplitude (AMP)

\begin{tabular}{|c|c|c|c|c|c|c|}
\hline Gênero/Espécie & $\mathrm{N}$ & MED & DP & \multicolumn{2}{|c|}{ AMP } & TOTAL \\
\hline & & \multicolumn{5}{|c|}{ Armadilha de Solo } \\
\hline Hololepta quadridentata** & 75 & 82,40 & 45,88 & $(0-$ & 42) & 1.030 \\
\hline Euspilostus (Hesperosaprinus) spp. ${ }^{\mathrm{ns}}$ & 75 & 17,84 & 15,35 & $(0$ & & 223 \\
\hline Euspilostus modestus" & 75 & $5.873,20$ & $3.891,19$ & $(3-$ & $68)$ & 73.415 \\
\hline \multirow[t]{2}{*}{ Carcinops Troglodytes ${ }^{\mathrm{ns}}$} & 75 & 776,24 & 402,21 & $(0-$ & 96) & 9703 \\
\hline & & \multicolumn{5}{|c|}{ Funil de Berlese-Tullgren } \\
\hline Hololepta quadridentata** & 75 & 3,36 & 2,74 & & & 42 \\
\hline Euspilostus (Hesperosaprinus) spp. ${ }^{\mathrm{ns}}$ & 75 & 0,96 & 1,14 & & & 12 \\
\hline Euspilostus modestus" & 75 & 54,08 & 44,78 & $(0-$ & 30) & 676 \\
\hline Carcinops Troglodytes ${ }^{\mathrm{ns}}$ & 75 & 315,44 & 160,02 & $(0-$ & 92) & 3.943 \\
\hline \multicolumn{7}{|l|}{ Covariáceis } \\
\hline TEMP $\left({ }^{\circ} \mathrm{C}\right)$ & 75 & 26,30 & 4,24 & 16 & 34 & - \\
\hline UMID (\%) & 75 & 66,15 & 20,29 & 24 & 100 & 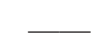 \\
\hline $\mathrm{PP}\left(\mathrm{mm}^{3}\right)$ & 75 & 139,89 & 117,16 & 0 & 341 & 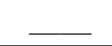 \\
\hline
\end{tabular}

\footnotetext{
* = Significativo $(\mathrm{P}<0,05)$ para índice pluvial;

** $=$ Significativo $(\mathrm{P}<0,01)$ para índice pluvial;

${ }^{\mathrm{ns}}=$ Significativo $(\mathrm{P}>0,05)$ para índice pluvial.
} 


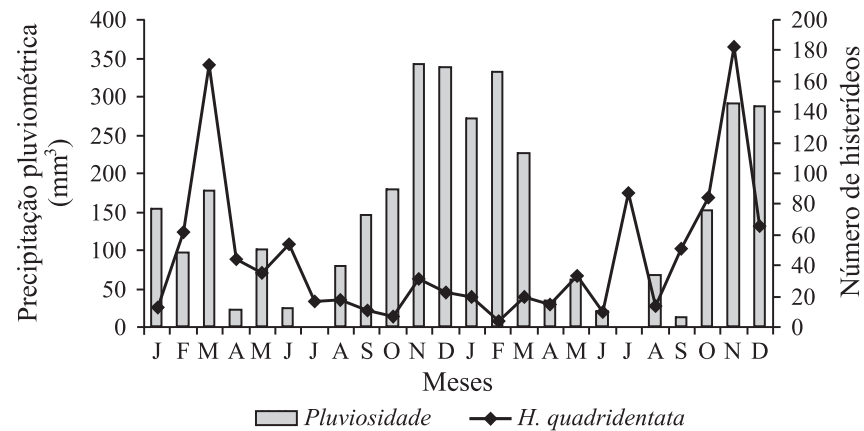

Fig. 1. Total de Hololepta quadridentata capturados em armadilhas de solo e funil de Berlese-Tullgren de janeiro de 2001 a dezembro de 2002, em granja avícola do estado de São Paulo.

Os resultados deste trabalho condizem com os dados obtidos por Gianizella \& Prado (1998), que coletaram sete espécies de histerídeos na granja Capuavinha em Monte Mor, SP, dentre as quais, E. modestus foi a mais abundante. Entretanto, são discordantes dos encontrados tanto por Bruno et al. (1993) quanto por Bicho et al. (2005), que concluíram que $C$. troglodytes foi o histerídeo predador mais numeroso. Tendo em vista que, E. modestus é mais abundante em coletas feitas por armadilhas de solo do que nas realizadas por funil de Berlese - Tulgren e que $C$. troglodytes mostrou o inverso, esta divergência nos resultados pode ter sido influenciado pelo método de amostragem adotado.

A explicação para a diferença entre o número e a proporção de cada espécie nos dois métodos de amostragem, talvez esteja nos hábitos das espécies, pois $C$. troglodytes $( \pm 3 \mathrm{~mm})$, parece ser mais ativo que $E$. modestus, conseguindo assim, cobrir maior área vertical no esterco e, conseqüentemente, ter mais chance de ser coletado por meio do funil de Berlese - Tullgren (Gianizella \& Prado 1998).

As análises de variância apresentaram resultados estatisticamente significativos no número total de coleópteros capturados nas diferentes coletas: E. modestus $(\mathrm{P}<0,05)$ e $H$. quadridentata $(\mathrm{P}<0,01)$ (Tabela I). Entretanto, Euspilostus

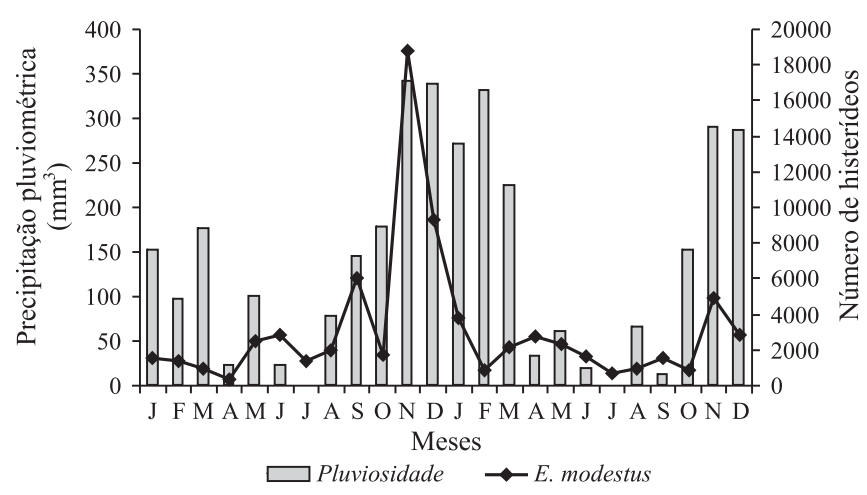

Fig. 2. Total de Euspilostus modestus capturados em armadilha de solo e funil de Berlese-Tullgren, de janeiro de 2001 a dezembro de 2002, em granja avícola do estado de São Paulo.
Tabela II. Coeficiente de correlação (momento-produto de Pearson) das covariáveis Temperatura (TEMP), Umidade relativa (UR) e Pluviometria (PP) para as diferentes espécies de histerídeos capturados em armadilhas de solo e funil de Berlese-Tullgren, em granja avícola do Estado de São Paulo.

\begin{tabular}{cccc}
\hline Gênero/Espécie & TEMP $\left({ }^{\circ} \mathrm{C}\right)$ & UMID $(\%)$ & $\mathrm{PP}\left(\mathrm{mm}^{3}\right)$ \\
\hline $\begin{array}{c}\text { Hololepta } \\
\text { quadridentata } \\
\text { Euspilostus }\end{array}$ & $-0,07144^{\mathrm{ns}}$ & $0,12528^{\mathrm{ns}}$ & $0,30722^{* *}$ \\
$\begin{array}{c}\text { (Hesperosaprinus) spp. } \\
\text { Euspilostus modestus }\end{array}$ & $-0,06179^{\mathrm{ns}}$ & $-0,32445^{\mathrm{ns}}$ & $0,220911^{\mathrm{ns}}$ \\
Carcinops troglodytes & $-0,12559^{\mathrm{ns}}$ & $-0,18312^{\mathrm{ns}}$ & $0,61690^{*}$ \\
& & & \\
Cas & $0,24686^{\mathrm{ns}}$
\end{tabular}

${ }^{*}=$ Significativo $(\mathrm{P}<0,05)$ para índice pluvial;

** = Significativo $(\mathrm{P}<0,01)$ para índice pluvial;

${ }^{\mathrm{ns}}=$ Significativo $(\mathrm{P}>0,05)$ para índice pluvial.

(Hesperosaprinus) spp. e C. troglodytes não foram encontradas diferenças estatísticas significativas $(\mathrm{P}>0,05)$ no número de indivíduo entre meses de coleta (Tabela I, II e Fig. 3). As correlações entre os totais de Euspilostus (H.) spp. e $C$. troglodytes encontrados nos diferentes métodos de coleta e os dados climáticos (umidade relativa e temperatura), foram de baixa magnitude, indicando baixas associações com estas covariáveis (Tabela II).

O número de histerídeos coletados em acúmulo de esterco de aves varia entre as localidades. No Brasil, em granjas do estado de São Paulo, Bruno et al. (1993) coletaram oito espécies de histerídeos, enquanto Bicho et al. (2005) encontraram duas espécies no Rio Grande do Sul. Legner et al. (1975) coletaram cinco espécies em granja avícola na Califórnia do Norte, USA. Na África do Sul, Hulley (1983) coletou três espécies em três granjas avícolas, dentre estas, $C$. troglodytes.

Quando verificam-se histerídeos em estercos bovino, notase que as espécies podem variar tanto no Brasil Marchiori et al. (2001), no sul de Goiás, Rodrigues \& Marchini (1998), em São Paulo e Koller et al. (2002) no Estado de Mato Grosso do Sul, quanto no continente africano, (Legner et al. 1981).

Os índices de abundância obtidos neste trabalho e realizado por Gianizella \& Prado (1998) sugerem que as duas espécies de histerídeos predadores mais importantes do estado de São Paulo são: Euspilostus modestus e Carcinops troglodytes. Gray et al. (1999); Kaufman et al. (2000, 2001 e 2002); Tobin et al.(1999) e Tobin \& Pitts (1999) relatam que a espécie predadora mais abundante e importante dos Estados Unidos é Carcinops pumilio (Erichson).

A dominância das duas espécies durante os meses de coleta, indica um alto grau de competição para o aproveitamento dos recursos oferecidos naquele ecossistema (Krebs 1989). Portanto, esses histerídeos podem estar produzindo um grande impacto sobre as populações de larvas de dípteros sinantrópicos e poderão ser de extrema importância em um futuro manejo integrado de pragas em acúmulo de esterco de aves poedeiras no Brasil. Pode-se inferir, ainda, que como os histerídeos predadores possuem hábitos polífagos, exclui-se 


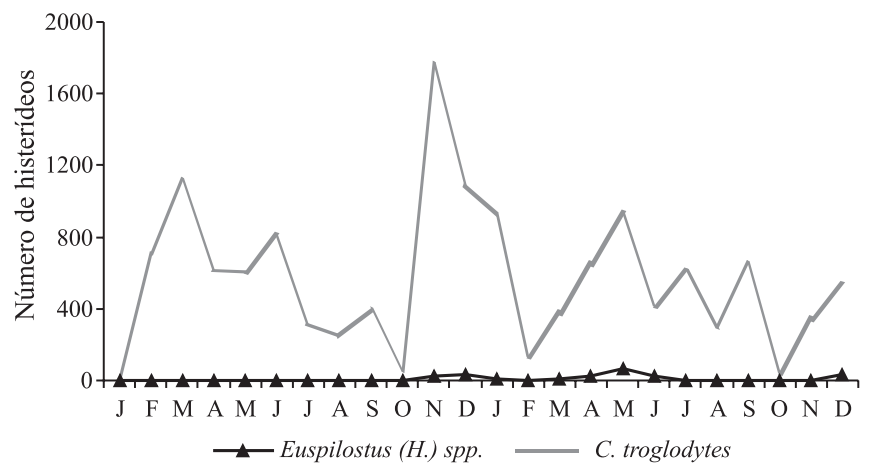

Fig. 3. Total de Euspilostus (Hesperosaprinus) spp. e Carcinops troglodytes capturados em armadilha de solo e funil de Berlese-Tullgren de janeiro de 2001 a dezembro de 2002, em granja avícola do estado de São Paulo.

a possibilidade de sua extinção em caso de extinção da presa, e, desta forma suas populações são mantidas em níveis aceitáveis.

De modo geral, E. modestus e H. quadridentata, mostraram relação direta com as variações climáticas, principalmente precipitação pluviométrica $(\mathrm{P}<0,05$ e $\mathrm{P}<0,01$, respectivamente), apresentando correlação positiva com esta covariável (Tabela II), quando o número de histerídeos aumentou significativamente nos meses que os índices pluviométricos se elevaram.

E. modestus e $H$. quadridentata podem ser classificadas como espécies unimodais por terem apresentado apenas um pico populacional durante o ano (Fig. 1 e 2) (Wolda 1988). Segundo Gianizella \& Prado (1998), a sazonalidade destas espécies dependem de um conjunto de fatores físico-químicos e biológicos, que determinam a melhor condição para o aumento populacional naqueles períodos do ano. É relevante ressaltar que, os picos populacionais das duas espécies não se sobrepõem o que pode ser considerado mecanismo de facilitação para evitar a competição entre os indivíduos das duas populações. Este trabalho demonstra que para o estudo populacional de histerídeos associados ao esterco de aves, deve ser feito por, pelo menos, dois métodos distintos de coletas.

Agradecimentos. À Dra Marisa Rossi Monteiro pela colaboração técnica, aos professores Maria Cândida de Oliveira Costa, Afonso Celso Navarro e Dr. Gilson Pereira de Oliveira pelas sugestões e comentários. Este trabalho foi financiado pelo Centro Universitário UNIFEOB.

\section{REFERÊNCIAS}

Arnett, Jr. R. H. 1968. Histeridae (Leach, 1815) pp. 369-384. In: The beetles of the United States, Ann Arbor, American Entomological Institute, 4th. ed., $1112 \mathrm{p}$.

Banzatto, D. A \& S. N. Kronca. 1989. Experimentação Agrícola. Ed. Funep, Jaboticabal, FCAV-UNESP, 247 p.

Bicho, C. L.; L. M. Almeida; P. B. Ribeiro \& P. Silveira Jr. 2005. Flutuação populacional circanual de coleópteros em granja avícola em Pelotas, RS, Brasil. Iheringia, Série Zoologia 95: 205-212. Borror, J. D. \& E. R. White. 1970. Insects. Boston: Houghton Mifflin Company.

Bruno, T. V.; A. M. M. Guimarães \& E. C. Tucci. 1993. Moscas sinantrópicas (Diptera) e seus predadores que se criam em esterco de aves poedeiras confinadas no Estado de São Paulo, Brasil. Revista Brasileira de Entomologia 37: 577-590.

Carraro, M. V. \& E. M. V. M. Azevedo. 2000. Controle alternativo contra pragas de interesse médico-veterinário e sanitário. Revista Brasileira de Medicina Veterinária 22: 11-13.

Clark, C. U. 1895. On the certain dung and carrion beetles. Journal of the New York. Entomological Society 3: 61.

Fowler, J. \& L.Cohen. 1990. Practical statistics for field biology. Philadelphia, Open University. Press., vii +225 p.

Georghiou, G. P. M.; M. K. Hawley \& E. C. Loomis. 1967. Pesticide resistance in the fly complex of California poultry ranches. California Agriculture 21: 9-11.

Gianizella, S. L. \& A. P. Prado. 1998. Levantamento e sazonalidade de coleópteros (Histeridae) em criação de aves poedeiras. Anais da Sociedade Entomológica do Brasil 27: 551-557.

Gray, J. P.; C. W. Maddox.; P. C. Toben.; J. D. Gummo \& C. W. Pitts. 1999. Reservoir competence of Carcinops pumilio for Salmonella enteritidis (Eubacteriales: Enterobacteriaceae). Journal of Medical Entomolgy 36: 888-891.

Guimarães, J. H. 1985. Moscas sinantrópicas: perspectivas de manejo integrado em aviários no Estado de São Paulo. Agroquímica 28: $19-24$.

Hinton, H. E. 1945. The Histeridae associated with stored products. Bulletin of Entomological Research 35: 309-340.

Hulley, P. E. 1983. A survey of flies breeding in poultry manure, and their potencial natural enemies. Journal of Entomological Society of South Africa 46: 37-47.

Imai, C. 1987. Control of insecticide resistance in a field population of housefiles, Musca domestica, by releasing susceptible flies. Research on Population Ecology 29: 129-146.

Kaufman, P. E.; S. J. Long.; D. A. Rutz \& C. S. Glenister. 2000. Preyand density-mediated dispersal in Carcinops pumilio (Coleoptera: Histeridae), a predator of house fly (Diptera: Muscidae) eggs and larvae. Journal of Medical Entomology 37: 929-932.

Kaufman, P. E.; S. J. Long.; D. A. Rutz \& C. S. Glenister. 2001. Larval production from field-collected Carcinops pumilio (Coleoptera: Histeridae) following three starvation periods. Journal of Medical Entomology 38: 278-281.

Kaufman, P. E.; D. A. Rutz \& J. K. Waldron. 2002. Seasonal variation in Carcinops pumilio (Coleoptera: Histeridae) dispersal and potential for suppression of dispersal behavior. Journal of Medical Entomology 39: 106-111.

Krebs, C. J. 1989. Ecological methodology. New York, Harper \& Row, xii +700

Koller, W. W.; A. Gomes \& S. R. Rodrigues. 2002. Fimicolous Histeridae Coleoptera in Campo Grande, MS, Brazil. Brazilian Journal of Biology 62: 473-478.

Legner, E. F. \& H. W. Brydon. 1966. Suppression of dung inhabiting fly population by pupal parasites. Annals of Entomological Society of America 59: 638-651.

Legner, E. F. \& G. S. Olton. 1968. Activity of parasites from Diptera: Musca domestica, Stomoxys calcitrans and species of Fannia, Muscina and Ophyra. Annals of Entomological Society of America 61: 1306-1314.

Legner, E. F.; G. S. Olton.; R. E. Eastwood \& E. J. Dietrick. 1975. Seasonal density distribution and interactions of predatory and scavenger arthropods in accumulating poultry wastes in coastal and interior Southern California. Entomophaga 20: 269-283.

Legner, E. F.; D. J. Greathead \& I. Moore. 1981. Equatorial east African predatory and scavenger arthropods in bovine excrement. Environmental Entomology 5: 620-625.

Marchiori, C. H.; A. T. Oliveira.\& A. X. Linhares. 2001. Artrópodes associados a massas fecais bovinas no Sul do Estado de Goiás. Neotropical Entomology 30: 19-24.

Mijares, A. S.; R. P. Pacheco \& S. H. Martinez. 2000. Susceptibilidade 
de las larvas de Aedes aegypti al parasitismo por Romanomermis culicivorax em condiciones de laboratorio y de campo em Oaxaca, México. Revista Panamericana de la Salud Publica 8:129_ 137.

North, M. O. \& D. D. Bell. 1990. Commercial Chicken Production Manual. New York, 4 ed., Van Nostrand Reihnold. 422 p.

Peck, J. H. \& J. R. Anderson. 1969. Arthropod predators of immature Diptera developing in poultry droppings in Northern California. Part I. Determination, seasonal abundance and natural cohabitation with prey. Journal of Medical Entomology 6: 163-167.

Peck, R. F. \& J. R. Anderson. 1970. Influence of poultry manure removal schedules on various diptera larval and selected arthropod predators. Journal of Economical Entomology 63: 82-90.

Rodrigues, S. R \& L. C. Marchini. 1998. Espécies de Histeridae coletadas em Piracicaba/SP. Scientia Agricola 55: 1-7.

SAS Institute Inc., 1995. SAS/STAT USER's guide: Basic and statistic. Cary, North Carolina, USA.

Summerlin, J. W. 1989. Techiques for collecting, rearing and handling histerid beetles. Southwestern Entomologist 14: 415-425.

Taylor, R. N. 1982. Insecticide resistance in houseflies from the Middle East and North Africa with notes on the use of various biossay techniques. Pesticide Science 13: 415-425.

Tobin, P. C.; S. J. Fleischer \& C. W. Pitts. 1999. Spatio-temporal dynamics of resistance and immigration populations of Carcinops pumilio (Coleoptera: Histeridae) in high-rise poultry facilities. Journal of Medical Entomology 36: 568-577.

Tobin, P. C \& C. W. Pitts. 1999. Flotation methods for extracting insects from poultry manure samples. Journal of Medical Entomology 36: 121-123

Walker, R. L. 1985. A pitffal trap study on the Carabidae and Staphylinidae (Coleoptera) in Country Durham. Entomology Monthly Magazin 121: 1-18.

Wenzel, R. L. 1955. The histerid beetles of New Caledonia (Coleoptera, Histeridae). Fieldiana: Zoology 37: 601-634.

Wolda, H. 1988. Insect seasonality: Why? Annual Review of Ecology and Systematics 19: 1-18. 